\title{
Retrospective analysis of efficacy of washed husband semen IUI for oligoasthenoteratospermia in an urban centre
}

\author{
Shanthi Sivaraman, Georgy Joy Eralil*
}

Department of Obstetrics and Gynecology, Sree Narayana Institute of Medical Sciences, Kerala, India

Received: 18 December 2018

Accepted: 11 January 2019

\section{*Correspondence:}

Dr. Georgy Joy Eralil,

E-mail: georgyeralil@yahoo.co.in

Copyright: () the author(s), publisher and licensee Medip Academy. This is an open-access article distributed under the terms of the Creative Commons Attribution Non-Commercial License, which permits unrestricted non-commercial use, distribution, and reproduction in any medium, provided the original work is properly cited.

\begin{abstract}
Background: The true incidence of male subfertility is unknown due to great variability in the prevalence of subfertility. Artificial insemination with husband's semen is the most widely used treatment for male infertility, usually presumed because of oligospermia, and for what is called 'mucus hostility' when there is failure of sperm penetration of cervical mucus despite normal seminal analysis.

Methods: The study was conducted in 438 couples with male factor infertility at the ARTC (artificial reproductive technique centre) of Sri Ramakrishna Hospital, Coimbatore. Results of at least two seminograms (based on WHO norms) were used to primarily classify males into three categories-oligozoospermic, asthenozoo spermic and oligoasthenoteratospermic. The media used were the Earle's Balanced Salt Solution (EBSS), Ham's F10 and Medicult. EBSS and Ham's F10 were obtained as "readymade" solutions from Sigma, USA. Medicult was imported from Denmark. EBSS and Ham's F10 were supplemented with protein using FCS (Fetal cord Serum) or HEPES (4(2hydroxyethyil)-1-piperazineethanesulfonic acid). Benzyl pencillin, 60mg per litre and Streptomycin, 50mg per litre were also to the media.

Results: By the DMRT analysis of post wash count, the influence of the count below 5 million or above 20 million on the pregnancy rate was significant at all the levels of male factor.

Conclusions: Considering the male factor, in cases of oligoasthenoteratospermia, IUI has a positive significant effect on the success rate of pregnancy at all three levels of the post wash sperm count.
\end{abstract}

Keywords: Intrauterine insemination, Oligoasthenoteratospermia, Sperm wash

\section{INTRODUCTION}

The true incidence of male subfertility is unknown due to great variability in the prevalence of subfertility. There is geographical variation, i.e. lowest incidence in the Middle East and highest incidence in Central Africa. There are also marked variations from region to region. Globally as an average factor in the male causing subfertility account for approximately $30 \%$ of all cases of subfertility, whilst in $40 \%$ of cases, both male and female factors are present. ${ }^{1}$ Infertility is a diagnosis that can have a considerable psychological impact to the man concerned, his partner and their relationship. Research has shown increased level of anxiety, depression, paranoia, compulsion, and even psychosis in both partners. This explains why, until recently, denial was the most frequent approach to the problem.

A quagmire of epidemiology, lifestyle, environmental pollution, systemic illnesses, medications, gene polymorphism and partner's fertility can all affect male fecundity. ${ }^{2}$ Artificial insemination with husband's semen is the most widely used treatment for male infertility, usually presumed because of oligospermia, and for what 
is called 'mucus hostility' when there is failure of sperm penetration of cervical mucus despite normal seminal analysis. $^{3}$

The aim and objectives of the study are:

The efficacy of sperm wash/IUI in couples with male factor infertility such as:

- Oligozoospermia

- Asthenozoospermia

- Oligoasthenozoospermia.

The success rate of IUI when the post wash sperm counts in three ranges:

- Below 5 million

- Between 5 and 20 million

- Above 20 million.

The success rate of IUI when categorizing the female partner into three age groups:

- Below 25 years

- Between 26 and 34 years

- At or above 35 years.

\section{METHODS}

The study was conducted in 438 couples with male factor infertility at the ARTC (artificial reproductive technique centre) of Sri Ramakrishna Hospital, Coimbatore.

Results of at least two seminograms (based on WHO norms) were used to primarily classify males into three categories-oligozoospermic, asthenozoospermic and oligoasthenoteratospermic.

The media used were the Earle's balanced salt solution (EBSS), Ham's F10 and Medicult. EBSS and Ham's F10 were obtained as "readymade" solutions from Sigma, USA. Medicult was imported from Denmark. EBSS and Ham's F10 were supplemented with protein using FCS (Fetal cord Serum) or HEPES (4(2-hydroxyethyil)-1piperazineethanesulfonic acid). Benzyl pencillin, 60mg per litre and Streptomycin, 50mg per litre were also added to the media.

\section{Preparation of FCS (fetal cord serum)}

1. Fetal cord blood $(20-30 \mathrm{ml})$ as collected aseptically in a bottle and centrifuged before it was allowed to clot. The plasma was placed in another sterile tube $(10 \mathrm{ml}$ Falcon tube).

2. When the plasma clotted after 1 hour at 37 degrees $\mathrm{C}$, the clot was removed using a $1 \mathrm{ml}$ pipette and the serum centrifuged to remove any fibrin or red blood cells.
3. The serum was the inactivated by heat, by placing it in $10 \mathrm{ml}$ tubes in a multiblock heater maintained at 56degrees C For 45 minutes. The serum was the cooled to room temperature and filtered into sterile tubes using $0.22 \mathrm{U}$ filter (sterivex).

4. After using the required volume to prepare the media for the patient, the excess serum was stored at 4degree $\mathrm{C}$ for 1 week or at -20 degrees $\mathrm{C}$ for longer periods.

The tubes, pipettes and other disposable plastic wares were obtained from the Falcon, or Nunc Company, USA. On the day of IUI, the male was advised to collect the ejaculate in a separate collection room. Labels were immediately placed for identification.

Once the specimen liquefied, sperm count and motility were tested and the remaining specimen was mixed with the media in the ratio of $1: 1$. The specimen was then centrifuged at 200-300rpm, for 6 minutes. The supernatant was discarded, and the pellet taken. Sperm preparation was done either by the "Swim-up," "Percoll," or "Sedimentation methods".

\section{Pallet and swim}

1. The semen and medium were mixed and centrifuged once.

2. All the supernatant was carefully removed and then $0.75 \mathrm{ml}$ of medium was very gently pipette over the pellet, taking care not to disturb it.

3. The sperm was allowed to swim up into the medium.

4. The supernatant was carefully removed from the pellet and placed in a clean centrifuge tube.

5. The sample was centrifuged again, resuspended in the medium, and assessed for sperm count and motility.

\section{Preparation of isotonic Percoll}

\section{Ingredients}

$10 \mathrm{X}$ saline $(9 \% \mathrm{NaCl})$ or $10 \mathrm{X} \mathrm{EBSS}-10 \mathrm{ml}$

Percoll solution- $90 \mathrm{ml}$

$5 \%$ human serum albumin- $9 \mathrm{ml}$

Sodium pyruvate- $3 \mathrm{ml}$

$60 \%$ sodium lactate $-0.37 \mathrm{ml}$

TM HEPES buffer- $2 \mathrm{ml}$

Gentamicin sulphate $5 \mathrm{mg} / \mathrm{ml}-2 \mathrm{ml}$.

The above ingredients were mixed and filtered through a 0.22 um Millipore filter.

\section{Preparation of the various gradient solutions}

\section{Two step gradient, $80 / 40$ solution}

$80 \%: 8 \mathrm{ml}$ isotonic saline $+2 \mathrm{ml}$ culture media $40 \%: 4 \mathrm{ml}$ isotonic saline $+6 \mathrm{ml}$ culture media. 


\section{Mini gradient solution}

$95 \%: 9.5 \mathrm{ml}$ isotonic saline $+0.5 \mathrm{ml}$ culture media $70 \%: 7 \mathrm{ml}$ isotonic saline $+3 \mathrm{ml}$ culture media $50 \%: 5 \mathrm{ml}$ isotonic saline $+5 \mathrm{ml}$ culture media.

1. $2-2.5 \mathrm{ml}$ of the $80 \%$ solution was pipette into the bottom of a conical centrifuged tube, and an equal volume of $40 \%$ solution was gently overlaid $2 \mathrm{ml}$ of the sample was layered on top of the $40 \%$ solution.

2. This was centrifuged at 200rpm for 20 minutes.

3. The pellet at the bottom of the $80 \%$ layer was carefully recovered and resuspended in $1 \mathrm{ml}$ of medium and the sample assessed for sperm count and motility.

4. If there were a high percentage of Immotile sperms, the solution was centrifuged at $200 \mathrm{rpm}$ for 5 minutes, the supernatant removed, $1 \mathrm{ml}$ of fresh medium was carefully layered over the pellet, and the motile sperms were allowed to swim up.

5. In the mini-gradient method, layers were made with $0.3 \mathrm{ml}$ of each solution: $95 \%, 70 \%$ and then $50 \%$. The semen was diluted with the media in a ratio of $1: 1$ and centrifuged at $200 \mathrm{rpm}$ for 10 minutes. The pellet was resuspended in $0.3 \mathrm{ml}$ culture media and layered over the mini gradient recovered and suspended in $0.5 \mathrm{ml}$ culture media, and the sample was assessed for sperm count motility.

\section{Sedimentation}

1. The semen was mixed with large volumes of the medium, the sample washed by dilution, centrifuged twice and the pellet recovered.

2. The pellet was resuspended in reduced volume of medium, so that the final motile sperm concentration did not become too dilute.

3. This final suspension was layered under paraffin oil (making one large droplet) in a small petridish. This was placed in a desiccator and gassed with $5 \%$ carbon dioxide.

4. The solution was left at Room Temperature (RT) for 3-24 hours. The motile spermatozoa; a longer period is usually more effective in reducing cells and debris but may also reduce the number of freely motile sperm in the upper part of the droplet.

5. The motile sperms were carefully aspirated by pipetting the upper part of the droplet.

The choice of sperm preparation method or combination of methods depends upon the assessment of:

- The motile count

- The ratio between motile and immotile count

- Volume of the sample

- Presence of antibodies, pus cells and debris.

Sperm samples which show moderate to high counts $(>35$ million motile sperms) with good forward progression could be prepared by the direct layering or swim up technique. This technique is also useful for viscous samples of when the total volume of semen is low. This method is not recommended when the motility is very poor or when there is a large degree of cellular contamination.

Discontinuous buoyant density gradient centrifugation is the method of choice for samples which show:

- Low motility

- Poor forward progression

- Large amount of debris and/or high number of cells

- Anti-sperm antibodies

- Teratospermia.

Discontinuous 2-3 step gradients are simple to prepare and highly effective in preparing motile sperm fractions from sub-optimal semen samples. The 3 layers of a mini gradient improve filtration and the smaller volumes improve recovery of sperm from severely oligozoospermic samples. Severely asthenozoospermic samples with a normal density and poor motility could also be distributed over a series of mini gradients.

The sedimentation method is useful for samples with very low counts and poor motility. It is very effective in removing debris but requires several hours of preparation time.

\section{Timing of insemination}

The female partners had controlled stimulated cycle with clomiphene citrate alone, Clomiphene plus hMG and hCG, or hMG and hCG. A vaginal ultrasound was done 2-3 days before the historical time of ovulation. Follicular size exceeds $18 \mathrm{~mm}$ and when the endometrial thickness was $8 \mathrm{mms}$, insemination was done. If hCG was used IUI was performed approximately 24-36 hours after its administration. Consecutive day insemination was not commonly used in cases of male factor infertility. However, when it was used, insemination was done 2448 hours after the first IUI.

\section{Insemination}

No chemicals were used for the preparation of the relevant body parts. Usually, the Jansen Anderson IUI catheter was used. In case of difficulty, an infant feeding tube-size $6 \mathrm{~F}$ was used. While performing the IUI, the catheter was advanced up to the fundus and slightly withdrawn. Utilizing $0.5 \mathrm{ml}$ of the sample, insemination was performed in an atraumatic fashion. Use of the tenaculum was seldom necessary. Following the insemination, the catheter was withdrawn for another centimeter and left in place for 1 minute. During this time, the uterine spasms which have been stimulated by the catheter or insemination would have subsided. The patient was asked to remain recumbent for 30 minutes. The typical patient received 3 cycles of IUI with CC (Clomiphene Citrate) stimulated ovulation, provided 
ovulatory function and endometrial development were appropriate. If pregnancy did not ensue. 1-2 cycles of Gn (Gonadotrophin) stimulated ovulation and IUI was attempted. The aggressiveness of the treatment increased as the duration of infertility was prolonged, if the woman was more than 35 years of age, or there was any pelvic or tubal pathology.

\section{Statistical analysis}

Data was entered into a database and analysed using IRRISTAT (International Rice Research Institute) Statistical Software. Results were summarized using percentages for categorical data and means for continuous data. Data was analysed by the Analysis of Variance (ANOVA) method with the female age as the relication factor. The response variable was the clinical pregnancy rate. The factors hypothesized were the male factor and the post wash count. Usually, ratios and percentages do not have a normal distribution; hence Arc sin transformation was done on the basic data. Interaction between the post wash count and the male factor was tested. The Duncan Multiple Range Test (DMRT)-mean comparison method was used to compare the averages of the male factor and post wash count. $\mathrm{P}$ value less than 0.05 was considered significant for all comparisons and no adjustment was made for multiple comparisons.

\section{RESULTS}

A total of 438 patients were included in this retrospective study. The success rate of sperm wash IUI was analysed with three variables:

- The male factor category

- The post-wash sperm count

- The age of the female partner.

There were 59 positive pregnancies (clinical) out of a total of 918 cycles. (Table 1). The pregnancy rate per patient was $13.7 \%$. the pregnancy rate per cycles was $6.4 \%$ (Figure 1).

\section{Male factor}

Three categories were analysed:

1. Asthenozoospermia (M1) $(\mathrm{n}=277)$

2. Oligozoospermia (M2) $(\mathrm{n}=49)$

3. Oligoasthenozoospermia (M3) $(n=112)$.

The male factor had a significant influence on the successful pregnancy rate (Table 4). Notably, oligozoosoermia (M2) showed a borderline significance on success of pregnancy (Table 5).

\section{Post wash count}

Three categories in the post wash count were analysed:

1. Below 5 million (p 1)

2. Between 5-20 million (p2)

3. Above 20 million (p3).

Counts below 5 million and above 20 million had a significant variation in the success rate of pregnancy at all three classification levels of the male factor (Table 5). The post wash count category between 5-20 million sperms did not have a significant variation in the success rate of pregnancy at all three classification levels of the male factor.

\section{Age of the female partner}

Age of the female partner was analysed by dividing them into 3 categories:

1. Less than or equal to 25 years (r1)

2. 26-34 years (r2)

3. Equal to or more than 35 years (r3).

All three categories of female partner age had a significant influence on successful pregnancy rate as seen in Table 2.

Table 1: Demography.

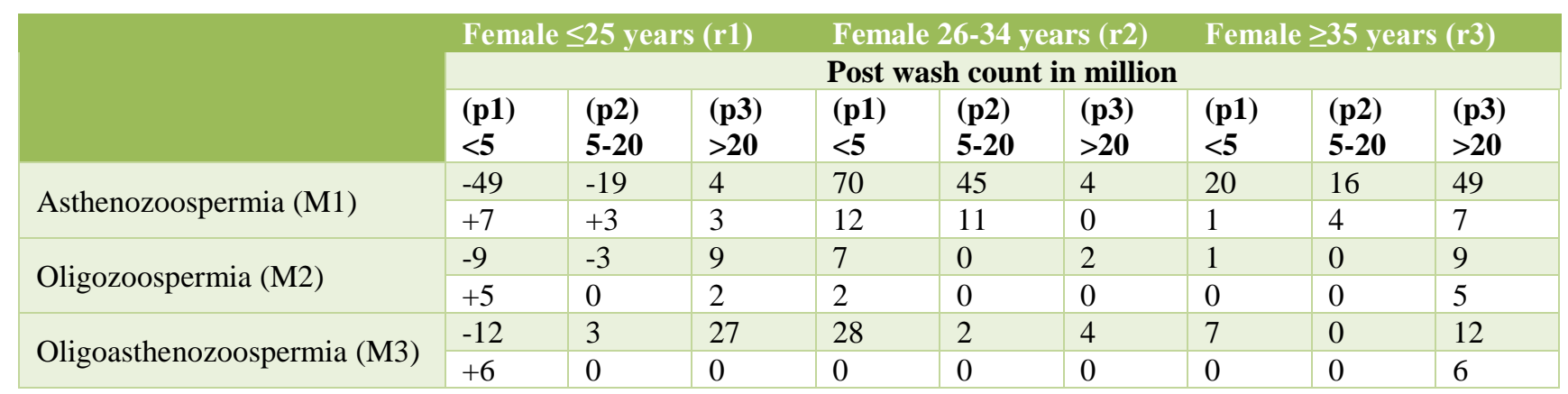

Total number of patients:438; Total number of cycles:918; Positive pregnancy rates:59; Pregnancy rate per patient:13.7\%; Response variable:positive pregnancy rate; Pregnancy rate per cycle:6.43\%; M1, M2, M3:three categories of male factor; p1, p2, p3:three levels of post wash count; r1, r2, r3:age of female partner (replication factor). 


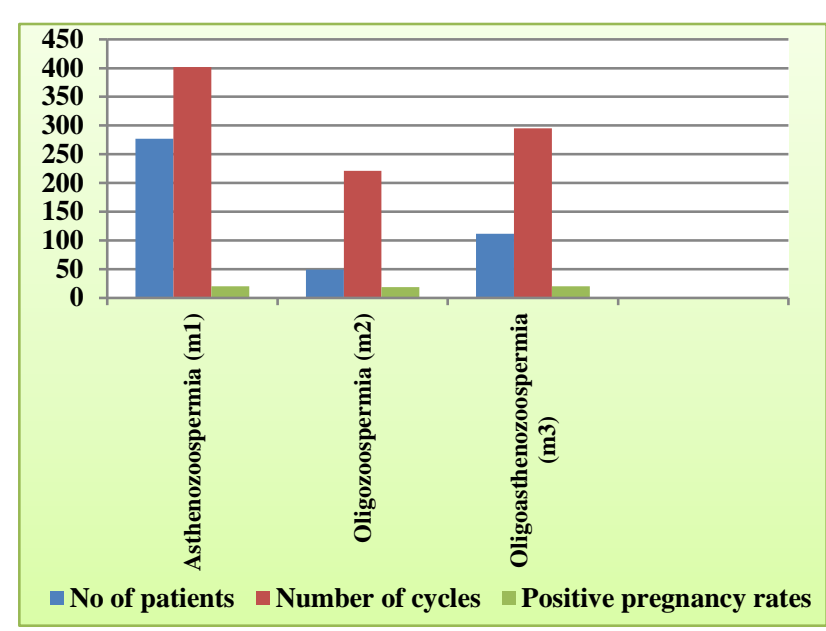

Figure 1: Demography.

Data was analysed by the analysis of variance (ANOVA) Method with the female age as the replication factor. The response variable was the clinical pregnancy rate. The factors hypothesized were the male factor and the post wash count (Table 2).

Table 2: Analysis of variance randomized complete block design-success ratio (pregnancy rate).

\begin{tabular}{|l|l|l|l|l|}
\hline $\begin{array}{l}\text { Post wash } \\
\text { count }\end{array}$ & Male & \multicolumn{4}{c}{ Age of female } \\
\hline \multirow{3}{*}{ factor } & r1 & r2 & r3 \\
\hline \multirow{2}{*}{$\mathrm{p} 2$} & M1 & 0.111 & 0.429 & 0.000 \\
\hline & M2 & 0.222 & 0.182 & 0.000 \\
\hline & M1 & 0.000 & 0.000 & 0.000 \\
\hline \multirow{2}{*}{ p3 } & M2 & 0.125 & 0.146 & 0.048 \\
\cline { 2 - 5 } & M3 & 0.333 & 0.000 & 0.000 \\
\hline \multirow{2}{*}{ REP Total } & M1 & 0.136 & 0.196 & 0.200 \\
\hline REP Mean & & 0.000 & 0.000 & 0.000 \\
\hline & M3 & 0.000 & 0.000 & 0.000 \\
\hline
\end{tabular}

Replication $(\mathrm{R})=3$; Treatment $=3 \times 3$; M1， M2, M3:three categories of male factor; p1, p2, p3:three levels of post wash count; $r 1, r 2$, r3:age of female partner (replication factor).

Usually, ratios and percentages do not have a normal distribution; hence Arc sin transformation was done on the basic data. According to ANOVA, age of the female partner was a significant factor on the successful pregnancy rate (Table 3).

By the Arc sin transformation, the ratio obtained in case of male factor was more than the F critical, and hence the male factor as an affecting factor on the pregnancy rate was significant (Table 4). By the same transformation in case of the post wash count, the $\mathrm{F}$ ratio obtained was less than the F critical, and hence the post wash count as an affecting factor on the pregnancy rate was not significant (Table 4). As per the data, the interaction (PxM) between the post wash count and the male factor was not significant.

Table 3: Success ratio (pregnancy rate).

\begin{tabular}{|l|l|l|l|l|}
$\begin{array}{l}\text { Post } \\
\text { wash } \\
\text { count }\end{array}$ & $\begin{array}{l}\text { Male } \\
\text { factor }\end{array}$ & $\mathbf{r 1}$ & $\mathbf{r}$ & $\mathbf{r}$ \\
\hline \multirow{2}{*}{$\mathrm{p} 1$} & M1 & 1.90925 & 3.75544 & 0.00000 \\
\hline & M2 & 2.70059 & 2.44506 & 0.00000 \\
\hline \multirow{2}{*}{ p2 } & M3 & 0.00000 & 0.00000 & 0.00000 \\
\hline \multirow{2}{*}{ M1 } & M2 & 3.02613 & 2.18980 & 1.25539 \\
\hline \multirow{2}{*}{ p3 } & M3 & 3.30815 & 0.00000 & 0.00000 \\
\hline REP Total & M2 & 2.11344 & 2.53742 & 2.56319 \\
\hline REP Mean & 0.00000 & 0.00000 & 0.00000 \\
\hline
\end{tabular}

M1, M2, M3:three categories of male factor; p1, p2, p3:three levels of post wash count; r1, r2, r3:age of female partner (replication factor).

By the Duncan Multiple Range Test (DMRT)-mean comparison method, the averages of the male factor and post wash count were compared. (Tables 5 and 6).

Through the ANOVA method indicates insignificance of the post wash count as an affecting factor on the pregnancy rate, the DMRT method showed that oligospermia alone (M2) indicated a significant variation on the success rate for a change of post wash count (Table 6) while the asthenospermia and oligoasthenospermia categories were on par (Table 6).

Table 4: Analysis of variance randomized complete block design.

\begin{tabular}{|l|l|l|l|l|}
\hline SV & DF & SS & MS & \\
\hline Age $(\mathrm{R})$ & 2 & 8.73070745 & 4.36535373 & $4.06 *$ \\
\hline Treatment & 8 & 22.84331038 & 2.85541380 & $2.66 *$ \\
\hline Post wash $(\mathrm{P})$ & 2 & 3.29117795 & 1.64558897 & $1.53 \mathrm{~ns}$ \\
\hline Male factor $(\mathrm{M})$ & 2 & 12.58445601 & 6.29222800 & $5.85 *$ \\
\hline P x M & 4 & 6.96767643 & 1.74191911 & $1.62 \mathrm{~ns}$ \\
\hline Error & 16 & 17.320605727 & 1.07537858 & \\
\hline Total & 26 & 48.78007511 & & \\
\hline
\end{tabular}

*= Significant at 5\% level; ns=not significant. 
Table 5: P x M table of means for success ratio based on back-transformed scale (average over 3 replications).

\begin{tabular}{|l|l|l|l|l|}
\hline Post wash $(\mathrm{p})$ & M1 & M2 & M3 & M-mean \\
\hline p1 & $0.1086 \mathrm{a}$ & $0.0896 \mathrm{ab}$ & $0.0000 \mathrm{a}$ & $0.0661 \mathrm{a}$ \\
\hline p2 & $0.1013 \mathrm{a}$ & $0.1270 \mathrm{a}$ & $0.0370 \mathrm{a}$ & $0.0884 \mathrm{a}$ \\
\hline p3 & $0.1760 \mathrm{a}$ & $0.0000 \mathrm{~b}$ & $0.0000 \mathrm{a}$ & $0.587 \mathrm{a}$ \\
\hline M-mean & 0.1286 & 0.0722 & 0.0123 & 0.0711 \\
\hline
\end{tabular}

Table 6: P x M table of means for success ratio based on back-transformed scale (average over 3 replications)-male factor $(\mathbf{M})$.

\begin{tabular}{|l|l|l|l|l|}
\hline Male factor $(\mathbf{M})$ & $\mathbf{m 1}$ & $\mathbf{m}$ & $\mathbf{m 3}$ & Male factor $(\mathbf{M})$ \\
\hline M1 & $0.1086 \mathrm{a}$ & $0.1013 \mathrm{a}$ & $0.1760 \mathrm{a}$ & $0.1286 \mathrm{a}$ \\
\hline M2 & $0.0896 \mathrm{a}$ & $0.1270 \mathrm{a}$ & $0.0000 \mathrm{~b}$ & $0.0722 \mathrm{ab}$ \\
\hline M3 & $0.0000 \mathrm{a}$ & $0.0370 \mathrm{~b}$ & $0.0000 \mathrm{~b}$ & $0.0123 \mathrm{~b}$ \\
\hline m-mean & 0.0661 & 0.0884 & 0.0587 & 0.0711 \\
\hline
\end{tabular}

In a column, means followed by common letter are not significantly different at the $5 \%$ level by DMRT method. By the DMRT analysis of post wash count, the influence of the count below 5 million or above 20 million on the pregnancy rate was significant at all the levels of male factor (Table 6).

\section{DISCUSSION}

The success rate of $\mathrm{Al}$ varies with its indication and ensuing pregnancies are not always easily attributable to the procedure. The overall success of IUI varies, with pregnancy rates ranging from as low as $2.7 \%$ to as high as $66 \% .^{4}$ Semen analysis is the first step to accurately diagnose male infertility. Sperm count, sperm motility and the percentage of sperm with normal morphology are the main criteria for the quality of semen. When determining sperm quality, the values established by the World Health Organization (WHO) 2010 are often used (however, the cut-off values for TPMSC (total motile progressive sperm count) are still controversial and vary between $0.3 \times 106$ and $20 \times 106 .^{5,6}$

Generally, comparison of data is difficult since often, when multifactorial infertility is treated by $\mathrm{Al}$, equivocal indications are sometimes. Used and data are not always completely reported. Cases of severe oligozoospermia (less than 10 million sperms per $\mathrm{ml}$ ), asthenozoospermia (less than $50 \%$ motility) of teratospermia (less than $50 \%$ normally configured sperm) have rarely produced pregnancy at rates which might be considered beyond the realm of chance. However, if the quality of semen is improved before insemination, treatment success rates are considerably higher.

By means of IUI, the cervix is bypassed, and seminal fluid is injected directly into the uterine cavity. Bypassing the cervical barrier with IUI results in significantly higher pregnancy rates than does intra cervical insemination
(ICI) in most published studies. However, some studies have reported little or no difference between the two. These discrepancies were due to lack of objective analysis of pregnancies resulting from IUI. As in any medical procedure, the end result used to measure success (e.g. Pregnancy is based on a series of interactions, each of which may have a number of variables affecting them. Often overlooked is the fact that many couples who entered into the IUI study protocols might have had previous cycles of treatment, and varied durations of infertility. There are notable differences in the literature as to the method of sperm preparation, the timing of insemination, the number of inseminations per cycle, the clinical indications for IUI and the presence or absence of agents that cause multiple follicular development. In addition, studies often fail to identify types of pregnancy-clinical or biochemical, ongoing or term. Reviewing the available literature, it can be expected that the highest success rates after IUI would be experienced by couples with cervical factor or male factor. authors compared the results of IUI with the volumes $0.3-0.4 \mathrm{~mL}$ and $0.5 \mathrm{~mL}$, authors detected no advantages. $^{7}$

Men who have severe sperm problems identified on their basic semen analysis are not good candidates for IUI. However, authors found that none of the basic semen parameters of concentration, motility or morphology was predictive of IUI success. ${ }^{8}$

The effectiveness of treatment of infertility disorders is assessed by the pregnancy rate per treatment cycle or per patient. The interpretation of these results with respect to IUI is clearly handicapped by a control group. To interpret the literature, some generalizations are made. Although cycle fecundity clearly indicates the degree of success of that treatment, it is known that even with abnormal sperm counts, spontaneous pregnancy may occur. It can also be safely assumed that the probability 
of conception is dependent on the underlying disorders. ${ }^{9}$ When comparing the semen analysis parameters between the two outcome groups, authors found that the initial motile and progressive sperm count and post motile and progressive sperm count was higher in the pregnant and not pregnant groups, whereas the post motile sperm count has no significant difference between groups. ${ }^{10}$

\section{Male factor}

Published literature shows that the overall pregnancy rate for all couples with male factor infertility is $21.7 \%$ (range of $0-57 \%$ ) and pregnancy rate per treatment cycle is $6.4 \%$ (range of $0-20.5 \%$ ) The result of the present study falls within this range and rate similar to the results seen in literature. Ovulatory cycles in which Gn (Gonadotrophin) or CC (Clomiphene Citrate) were used did not appear to significantly improve the pregnancy rate, per couple or per treatment cycle. In the present study, oligospermia had a significant influence on the successful pregnancy rate in all categories of post wash count. Similarly, post wash counts of less than 5 million and more than 20 million also had a significant effect on the pregnancy rate. $^{11}$

IUI has been used empirically in the past for the treatment of various types of infertility. "Male subfertility" has been the most controversial indication for IUI. Although the pregnancy rate of IUI in the presence of male factor infertility is low. The procedure should not be rejected outright without considering the cost effectiveness when compared with GIFT (gamete intrafallopian tube transfer) and IVF (in-vitro fertilization). In case of male factor infertility, when more than 1 million motile sperms can be harvested, IUI should provide a higher pregnancy rate (especially with super ovulation) than the pregnancy rate that would occur spontaneously. ${ }^{12}$ The recommendations are that, no more than 6 cycles should be attempted. The maximum 60 -minute limit of the interval between the onset of postwash sperm incubation and IUI time may increase pregnancy rates. ${ }^{13}$

There are still many questions regarding IUI, but the use of the technique has been firmly established. This technique should continue to be a safe, noninvasive procedure that can be used to treat infertility in most couples trying to achieve pregnancy.

\section{CONCLUSION}

The conclusion drawn from this study involving three variables in the relationship between. Sperm wash/intra uterine insemination (IUI) and the success rates of pregnancy (as indicated by clinical pregnancy) are as follows:

\section{Impact of the male factor}

Three categories of male factor infertility were studied:
1. Oligozoospermia

2. Asthenozoospermia

3. Oligoasthenozoospermia.

Considering the male factor, in cases of oligozoospermia, IUI has a positive significant effect on the success rate of pregnancy at all three levels of the post wash sperm count.

\section{Impact of the post wash sperm count}

Three categories of post wash sperm count studied:

1. Below 5 million

2. Between 5-20 million

3. Above 20 million.

Considering the post wash sperm count below 5 million and above 20 million have a significant impact on the success rate of pregnancy after IUI at all the three classification levels of male factor infertility.

\section{Impact of the female partner's age}

Three categories of age groups to which the female partner belongs to were studied:

1. Less than 25 years

2. Between 26 and 35 years

3. Above 35 years.

IUI has a significant impact on the success rate of pregnancy at all 3 categories of the age of the female partner, in the three classification levels of male factor infertility.

\section{Funding: No funding sources \\ Conflict of interest: None declared}

Ethical approval: The study was approved by the Institutional Ethics Committee

\section{REFERENCES}

1. Kumar N, Singh AK. Trends of male factor infertility, an important cause of infertility: A review of literature. J Hum Reprod Sci. 2015;8(4):191-6.

2. Olsen J, Ramlau-Hansen $\mathrm{CH}$. Epidemiologic methods for investigating male fecundity. Asian $\mathbf{J}$ Androl. 2014;16:17-22

3. Vasan SS. Semen analysis and sperm function tests: How much to test?. IJU. 2011;27(1):41-8.

4. Zadehmodarres S, Oladi B, Saeedi S, Jahed F, Ashraf $H$. Intrauterine insemination with husband semen: an evaluation of pregnancy rate and factors affecting outcome. J Assist Reprod Genet. 2009;26(1):7-11.

5. World Health Organization, Department of Reproductive Health and Research. WHO laboratory manual for the examination and processing of human semen, Switzerland, WHO Press, 2010. 
6. Van Weert JM, Repping S, Von Voorhis BJ, Bossuyt PMM, Mol BWJ. Performance of the post-wash total motile sperm count as a predictor of pregnancy at the time of intrauterine insemination: a meta-analysis. Fertil Steril. 2004;82:612-20.

7. Koyun OK. Post-wash semen and pregnancy outcomes. J Turkish-German Gynecol Assoc. 2013;14:142-5.

8. Branigan E, Estes A, Walker K. The effect of processed total motile sperm counts and twenty four hour sperm survival on the efficacy of intrauterine insemination in male infertility. Andrology. 2017;6:191.

9. Zhang E, Tao X, Xing W, Cai L, Zhang B. Effect of sperm count on success of intrauterine insemination in couples diagnosed with male factor infertility. Mater Sociomed. 2014;26(5):321-3.

10. Tan O, Ha T, Carr BR, Nakonezny P, Doody KM, Doody KJ. Predictive value of postwashed total progressively motile sperm count using CASA estimates in 6871 non-donor intrauterine insemination cycles. J Assist Reprod Genet. 2014;31(9):1147-53.
11. Lemmens L, Kos S, Beijer C, Brinkman JW, van der Horst FA, van den Hoven L, et al. Predictive value of sperm morphology and progressively motile sperm count for pregnancy outcomes in intrauterine insemination. Fertil Steril. 2016;105(6):1462-8.

12. Badawy A, Elnashar A, Eltotongy M. Effect of sperm morphology and number on success of intrauterine insemination. Fertil Steril. 2009;91(3):777-81.

13. Koyun E, Okyay RE, Doğan OE, Kovalı M, Doğan SS, Gülekli B. The effect of intrauterine insemination time on semen parameters. J Turk Ger Gynecol Assoc. 2014;15(2):82-5.

Cite this article as: Sivaraman S, Eralil GJ.

Retrospective analysis of efficacy of washed husband semen IUI for oligoasthenoteratospermia in an urban centre. Int J Reprod Contracept Obstet Gynecol 2019;8:445-52. 